(C) 1985 IEEE. Personal use of this material is permitted. However, permission to reprint/republish this material

for advertising or promotional purposes or for creating new collective works for resale or redistribution to servers

or lists, or to reuse any copyrighted component of this work in other works must be obtained from the IEEE.

\title{
A NOVEL RF CAVITY TUNING FEEDBACK SCHEME FOR HEAVY BEAM LOADING
}

\section{F. Pedersen}

CERN, CH-1211 Geneva 23, Switzerland

\section{Abstract}

The beam-cavity instability threshold as given by the Robinson ${ }^{1}$ criterion for the open loop case is of ten improved by phase, amplitude and tuning loops. The improvement obtained is, however, limited by cross coupling as the transfer functions vary with the relative beam loading. A more powerful scheme is fast additive feedback around the final amplifier stage. With this scheme bean loading several orders of magnitude above the Robinson threshold have been achieved in the CERN ISR and AA in the absence of fast tuning loops. A novel scheme to include a tuning loop insensitive to relative beam loading is presented, and first test results from the application of this scheme to the CERN PS booster second harmonic cavity are given.

\section{Review of Robinson Stability Criterion for Beam-Cavity Interaction}

We Iimit ourselves in this context to the interactions of the fundamental components of the beam and generator currents with the cavity (steady state condition), small modulations of these quantities (stability), and sudden changes of these (transient beam loading).

$\operatorname{are}^{2}:$

The main parameters involved in this problem

$I_{B}$ : fundamental component of beam current. $I_{B}=2 I_{D C}$ in the limit of short bunches.

$\varphi_{B}$ : phase angle of $\bar{I}_{B}$. In the limit of short bunches $\varphi_{B}=\varphi_{S}$ (= stable phase angle).

$\bar{I}_{T}$ : total current injected into cavity. $\overline{\mathrm{I}}_{T}=\overline{\mathrm{I}}_{B}+\overline{\mathrm{I}}_{\mathrm{G}}$.

$\bar{V}$ : cavity peak gap voltage.

$\varphi_{Z}$ : cavity impedance phase angle, $\varphi_{Z}=\arg \left(z_{C}\left(j \omega_{0}\right)\right)$, at the operating RF frequency $\omega_{0}$. Phase angle between $\overline{\mathrm{I}}_{\mathrm{T}}$ and $\overline{\mathrm{V}}$.

$\overline{\mathrm{I}}_{\mathrm{G}}$ : generator current (transformed to gap).

$\varphi_{\mathcal{L}}$ : phase angle between generator current $\overline{\mathrm{I}}_{\mathrm{G}}$ and gap voltage $v$. Apparent impedance phase angle of beam and cavity load: loading phase angle.

$\bar{I}_{0}$ : generator current required to give the same gap voltage without beam load and with cavity tuned to resonance. Equal to shunt resistor current: $\bar{I}_{0}=\bar{V} / R$.

$Y$ : relative beam loading $Y=I_{B} / I_{0}$.

$P_{0}$ : cavity power dissipation, $P_{0}=V I_{0} / 2$.

$\mathrm{P}_{B}$ : active beam power, $\mathrm{P}_{\mathrm{B}}=\left(\mathrm{VI}_{\mathrm{B}} \sin \varphi_{B}\right) / 2\left(\mathrm{P}_{\mathrm{B}}\right\rangle 0$ for acceleration, ${ }^{P_{B}}$ ( 0 for deceleration).

0 : cavity damping rate, $a=1 /(2 \mathrm{RC})$

$R$ : cavity shunt impedance.

L : cavity equivalent inductance.

C : cavity equivalent capacity.

A necessary (but not sufficient) condition for stationary operation is given by the steady state relations imposed by the vector diagram in Fig. 1.
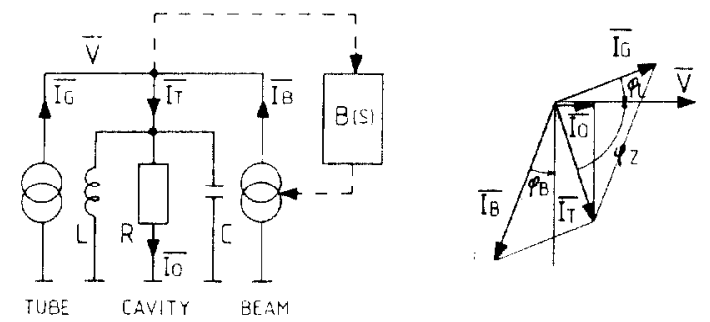

Fiq. 1 - Circuit equivalent and steady state vector diagram (below transition).
For a given $\bar{I}_{B}, \bar{v}$ and $\varphi_{Z}$, the required $I_{G}$ and $\varphi_{L}$ are:

$$
\begin{gathered}
\tan \varphi_{L}=\frac{I_{0} \tan \varphi_{Z}-I_{B} \cos \varphi_{B}}{I_{0}+I_{B} \sin \varphi_{B}}=\frac{\tan \varphi_{Z}-Y \cos \psi_{B}}{1+Y \sin \varphi_{B}} \\
I_{G}=\frac{I_{0}+I_{B} \sin \varphi_{B}}{\cos \varphi_{L}}=\frac{I_{0}\left(1+\sin \varphi_{B}\right)}{\cos \varphi_{L}}=2 \frac{P_{0}+P_{B}}{V \cos \varphi_{L}}
\end{gathered}
$$

Minimum generator current is obtained for $\varphi_{\mathrm{L}}=0$ (or $\left.\mathrm{n}\right)$ by detuning the cavity to obtain reactive compensation:

$$
\begin{aligned}
& \tan \varphi_{Z}=\left(\mathrm{I}_{\mathrm{B}} / \mathrm{I}_{0}\right) \cos \varphi_{\mathrm{B}} \\
& \mathrm{I}_{\mathrm{G}}=2\left(\mathrm{P}_{0}+\mathrm{P}_{\mathrm{B}}\right) / \mathrm{V} \\
& \varphi_{\mathrm{L}}=0\left(\text { or } 180^{\circ}\right)
\end{aligned}
$$

The stability can be determined by considering the propaqation of small amplitude and phase modulations of the vectors $1^{-2}$. If no feedback (tuning, AVC, or phase loop) relates $\overline{\mathrm{I}}_{G}$ to $\bar{v}$ or $\overline{\mathrm{I}}_{B}$, and only the beam transfer function relates $\bar{I}_{y}$ to $\bar{V}$, stability requires:

$$
0<\sin 2 \varphi_{2}<2 \cos \varphi_{B} / \Psi
$$

which is the Robinson criterion' below transition. Above transition the sign of $\varphi_{2}$ is inverted. The regions of stability are plotted in Fig. 2 versus $\phi_{2}$ toyether with the $\varphi_{2}$ required for reactive compensation (3) for $\varphi_{B}=0$ and $\left|\varphi_{B}\right|=30^{\circ}$.

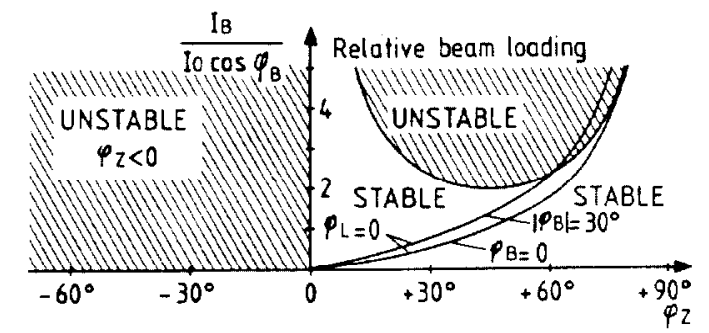

ric...2 - Stability versus impedance phase angle.

For $\varphi_{B}=0$ the system is theoretically stable tor any beam loading if $\varphi_{L}=0$, while for $\varphi_{B} \neq 0$ the $\mathfrak{w}_{L}=0$ curve will intercept the unstable area. By substituting the reactive compensation condition (3) into (4) the high current limit becomes:

$$
\begin{gathered}
\mathrm{Y}=\mathrm{I}_{\mathrm{B}} / \mathrm{I}_{\mathrm{O}}<1 /\left|\sin \mathrm{P}_{\mathrm{B}}\right| \\
\left|\mathrm{P}_{\mathrm{B}}\right|<\mathrm{P}_{0}
\end{gathered}
$$

so in this case stability requires that the power exchanyed with the beam is less than the cavity dissipation.

Since the tune is normally controlled to maintain $\varphi_{\mathrm{L}}$ near zero it may be useful to plot the stability limits versus the loading phase angle (Fig. 3). For $\varphi_{\mathrm{B}}=+30^{\circ}$, the beam power exceeds cavity power for $Y>2$, the instability limit for $\varphi_{L}=0$. Although this limit can be raised by increasing $\varphi_{L}$, and goes to infinity for $\Psi_{\mathrm{L}}>\varphi_{\mathrm{B}}$, the stability margin is insufficient in practice if the $P_{B}<P_{0}$ limit is exceeded. For $\varphi_{B}=$ 0 the high current limit is at infinity for $\varphi_{L}=0$ since the active beam power is zero. For $\varphi_{\mathrm{B}}=-30^{\circ}$, $\mathrm{P}_{\mathrm{B}}+\mathrm{P}_{0}\langle 0$ for $\mathrm{Y}>2$, and the loading phase angle must be in the range $-270<\varphi_{\mathrm{L}}<-90$ since the generator must absorb power from the beam-cavity system. Reactive compensation requires $\varphi_{L}=-180^{\circ}$. Most of this area with reversed generator power is unstable. 

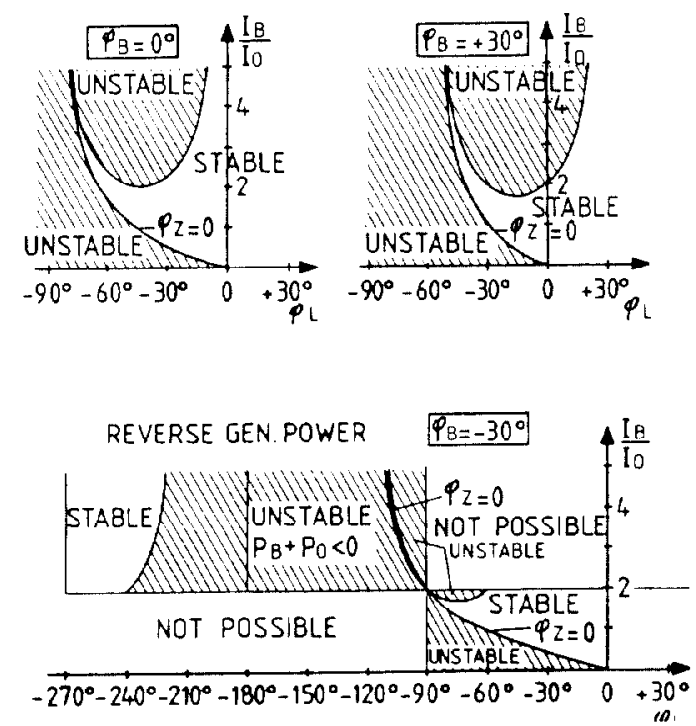

Fiq. 3 - Stability versus loading phase angle.

If a tuning feedback loop is added, the characteristic equation is of 5 th order. Nevertheless, an analytical stability criterion can still be given ${ }^{2}$. For a sufficiently slow tuning loop:

$$
\omega_{\mathrm{T}} / \omega_{\mathrm{S}} \ll 2 \omega_{\mathrm{S}} / \sigma \text { and } \omega_{\mathrm{T}} / 0 \ll 2
$$

where $\omega_{T}$ is the bandwidth of the tuning loop, this stability criterion approaches the Robinson criterion (4).

\section{Beam Loading Compensation Schemes}

\section{Phase and Amplitude Feedback}

In most proton accelerators, the fast sweep rate, relatively low $Q$ cavities, and low synchrotron frequency imply that the required tuning feedback loop does not fulfil the slow conditions $(6)$. In fact, if $w_{T} / \omega_{S}>2 w_{S} / 0$, the $\varphi_{2}-Y$ stability diagram becomes inverted with respect to the sign of $\varphi_{2}$, so the synchrotron dipole motion $(\mathrm{n}=0, \mathrm{~m}=1)$ is unstable for $\varphi_{\mathrm{L}}=$ 0 , even for low relative beam loading ${ }^{2}$. The additional strong damping from a beam-cavity phase feedback loop ensures stability for any $\varphi_{2}$, while an additional AVC feedback $100 \mathrm{p}$ controls the amplitude of the generator given by (2) to maintain the desired cavity voltage $v$ independent of varying beam loading. Minimum generator current is ensured automatically by the tuning loop, which controls the loading phase angle $P_{L}$ and thus ensures reactive compensation for a varying beam loading.

The beam-cavity interaction is thus strongly modified by the feedback loops, so the Robinson criterion (4) no longer applies. Experience and analysis 2 show, however, that stability is lost for $Y>2$ to 3 , a threshold comparable to the Robinson high current limit. Stability is lost due to the appearance of dominant crosscouplings between the feedback loops, partly due to the geometry of the vectors (Fig. 1), and partly due to the way phase and amplitude modulations are transmitted through the detuned cavity.

\section{Low Level Feed forward Compensation}

An RF signal proportional to $\overline{\mathrm{I}}_{B}$ is added in the low level drive chain such that it gives a contribution to $\overline{\mathrm{I}}_{G}$ equal to the beam $R F$ current $\overline{\mathrm{I}}_{B}$, but with opposite phase. The steady state conditions, (1) and (2), are nevertheless unchanged, so no extra RF power is needed. Only the way the $\overline{\mathrm{I}}_{\mathrm{G}}$ vector is controlled by the AVC and phase feedback loops is changed, resulting in a reduced crosscoupling from the vector geometry.
Experience and analysis show a dramatic increase in the instability threshold. This scheme has been successfully applied in the CERN PS and PSB. The instability threshold can probably be raised an order of magnitude. It is difficult to apply this scheme while the frequency is varying.

\section{Amplifier Feedback}

By subtracting a fraction of the RF gap voltage from the drive signal at an appropriate point in the amplifier drive chain (Fig. 4a), the effective cavity impedance as seen by the beam will be reduced a factor $(1+H)$, where $H$ is the feedback open loop gain. This is the most powerful scheme known and can be applied even for varying frequency. It has been used at the CERN ISRs $(9.5 \mathrm{MHz}$, fixed frequency, $H=60)$, CERN AA $^{6}(1.85 \mathrm{MHz}$, fixed frequency, $\mathrm{H}=120)$, and the CERN PSB $7(6-16 \mathrm{MHz}, H=5$ to 12$)$. It will be analyzed in a following paragraph.

\section{Hiqh Level_Feedforward Compensation}

The beam RF current from a pick-up is amplified and injected into the cavity with opposite phase of $\bar{I}_{B}$ by means of a separate $R F$ power amplifier capable of supplying the full beam current. The cavity tune is thus not used for reactive compensation. It is an expensive but powerful solution, applicable for fixed frequency only. It was added to the ISR RF system not so much to improve stability but due to a power limitation in the RE power amplifiers.

\section{Beam-Cavity Stability with Amplifier Feedback. Tuning}

A fraction $\beta$ of the cavity voltage $\bar{v}$ is subtracted from the drive signal $\bar{v}_{i}$ and amplified in the final power amplifier with transconductance $S$ (rig. 4a). The feedback open loop gain is $H=$ ASR. The summing point is transformed into two current sources (Fig. 4b):

$$
\bar{I}_{G}=S \bar{V}_{i}-\beta S R(\bar{V} / R)=\bar{I}_{G}^{k}-H \bar{I}_{0}
$$

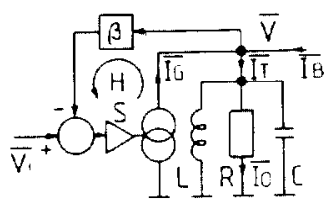

a)

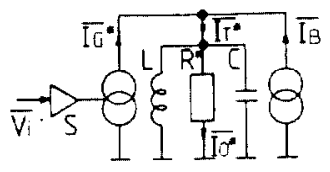

()

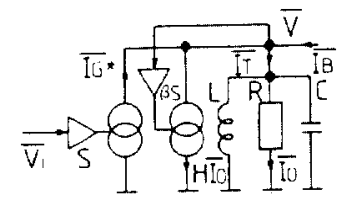

b)

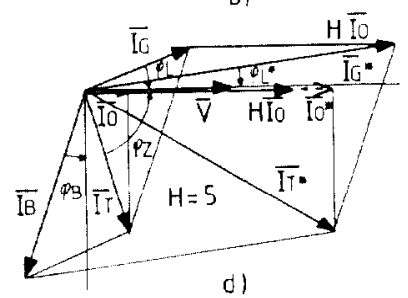

d)
Eig. 4 - Equivalent circuits with amplifier feedback.

The feedback current $H \vec{I}_{0}$ and the resistor current $\overrightarrow{\mathrm{I}}_{0}$ are combined as a new resistor current $\overrightarrow{\mathrm{I}}_{0}^{*}$ in a dynamically equivalent cavity circuit with no feedback (Fig. 4C):

$$
\begin{aligned}
& \overline{\mathrm{I}}_{0}^{*}=(1+\mathrm{H}) \overline{\mathrm{I}}_{0} ; \mathrm{R}^{*}=\mathrm{R} /(1+\mathrm{H}) \\
& \overline{\mathrm{I}}_{\mathrm{T}}^{*}=\overline{\mathrm{I}}_{\mathrm{T}}+\mathrm{H}_{0}=\overline{\mathrm{I}}_{\mathrm{G}}^{*}+\overline{\mathrm{I}}_{\mathrm{B}} \\
& \sigma^{*}=\sigma(1+\mathrm{H}) ; \mathrm{Y}^{*}=\mathrm{Y} /(1+\mathrm{H})
\end{aligned}
$$


This means that with respect to beam-cavity interactions and response to control modulations of $v_{i}$, the cavity with feedback behaves like a cavity with a shunt impedance and a beam loading a factor $(1+H)$ lower. The real and fictive vectors defined above are shown in $8 \mathrm{ig}$. $4 \mathrm{~d}$.

If no additional feedback is added, the Robinson criterion applies but with a high current limit raised by the feedback factor $(1+H)$. The low tuning bandwidth requirement may become more strict though, since $\sigma^{*}=\sigma(1+H)$.

If additional phase and amplitude feedback is applied to $\bar{v}_{i}$, stable feedback loops can usually be obtained up to $X^{*}<2$ or $Y<2(1+H)$. The same applies to the tuning loop if $\varphi_{L}^{*}$, the phase angle between $I_{G}^{*}$ (or $\bar{V}_{i}$ ) and $V$ is used as reference. However, a small exror in $\varphi_{L}^{x}$ or in the phase of $\beta$ produces a $(1+H)$ times larger error in $\varphi_{L}$ (if no beam load), which for high $H$ produces unacceptably large errors in $\varphi_{L}$, so it seems better to use $\varphi_{L}$. But as $I_{B}$ now can be very large with respect to $I_{0}, I_{G}$ varies strongly with $P_{B}$ resulting in large variations and change of sign of tuning loop gain (Fig. 5).

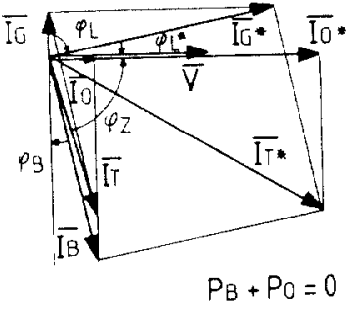

a)

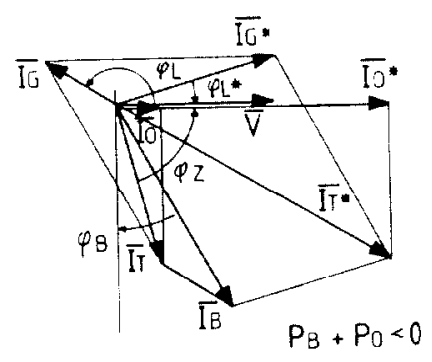

b)

\section{Fig. 5 - Steady state vector diagrams with zero and reverse generator power.}

A much better reference quantity is the reactive generator power $P_{Y}$. From (1) and (2) we get:

$$
P_{r}=\left(I_{G} V \sin \varphi_{L}\right) / 2=P_{0}\left(\tan \varphi_{Z}-Y \cos \varphi_{B}\right)
$$

We define the normalized detuning $x=\Delta w_{r} / 0=$ tanp $z$ and its value for reactive compensation $x_{0}=$ $Y \cos \psi_{\mathrm{B}}$. The normalized reactive generator power is:

$$
P_{r} / P_{0}=x-x_{0}
$$

yielding unity tuning gain independent of $I_{B}$ and $\varphi_{B}$. This is only the $D C$-response though. The dynamic response is obtained by relating $P_{r}$ to $Q_{L}^{*}$. The feedback current $H \bar{I}_{0}$ can be considered in phase with $\bar{V}$ over a wide bandwidh, so we get from Fig. Sd:

$$
\sin \varphi_{L}^{*}=\frac{I_{G} \sin \varphi_{L}}{I_{G}^{*}} \times \frac{I_{G} \sin \varphi_{L}}{(1+H) I_{0}}=\frac{1}{1+H} \frac{P_{r}}{P_{0}}
$$

The dynamic response is thus equivalent to $\varphi_{\mathrm{L}}^{\mathrm{R}}$, the loading phase of the transformed cavity equivalent circuit, so the tuning response is also well behaved since $\mathrm{Y}^{*}$ is low.

\section{Application to the CERN PS Booster second Harmonic Cavity}

Although high gain amplifier feedback is used in the CERN ISR 5 and CERN $A A^{5}$, no tuning loop is used in the $A A$ when the voltage is low or in the ISR. The second harmonic cavity in the CERN Booster?, however, uses an amplifier with wideband feedback and a tuning feedback loop to allow operation from 6 to $16 \mathrm{MHz}$. The active power delivered to the beam is small $\left(\varphi_{B}=0\right)$ but small phasing errors can cause large variations in $\mathrm{P}_{\mathrm{B}} / \mathrm{P}_{0}$ making the original phase loop, which used $\boldsymbol{p}_{\mathrm{L}}$ as reference, unstable.

The normalized reactive power signal is obtained by multiplying the gap signal $(\vec{V})$ shifted $90^{\circ}$ by the grid signal $\left(\vec{I}_{G}\right)$ (Fig. 6). The gap signal is normalized to a constant amplitude in an AVC amplifier, whose internal gain control voltage is used to control the gain of an identical amplifier in the grid chain so both amplifiers have identical gains, independent of $\bar{I}_{G}$ This takes care of the $P_{0}$ normalization.

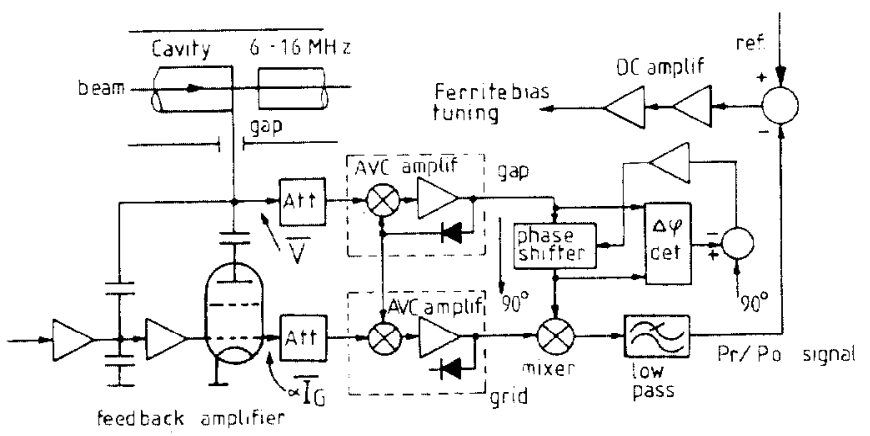
Fig. 6 - Tuning feedback loop using normalized
reactive powe.

The new tuning scheme makes the tuning loop insensitive to variations in $\mathrm{P}_{\mathrm{B}}$. Stable operation under reversed generator power conditions (Fig. 5b) has been observed.

The scheme is in particular useful where several cavities in one ring operates with high $I_{B} / 1_{U}$ and a net $\varphi_{B}$ near zero. Small variations in $\varphi_{B}$ from cavity to cavity can thus cause relatively large variations in $\mathrm{P}_{\mathrm{B}} / \mathrm{P}_{0}$.

\section{Acknowledgements}

I would like to thank $G$. Nassibian for his encouragement to carry out this work and for reading encouragement to carry out this work and assistance of J.M. Baillod in building the electronics is appreciated

\section{References}

[1] K.W. Robinson, CEA, Report no. CEAL1010, 1964.

[2] F. Pedersen, IEEE Trans, Nucl, Sci, NS22. p. 1906,1975 .

[3] D. Boussard, These Proceedings.

[4] D. Boussard, CERN SPS/ARF/DB/g5/78-16, 1978 .

[5] F.A. Ferger, W. Schnell, USSR 2nd Nat. Conf. on Particle Accelerators, Moscow, Sept. 1970

[6] R. Johnson et al., IEEE Trans. Nucl. Sci., NS-30, p. $2290,1983$.

(7) J.M. Baillod et al., IEEE Trans. Nucl. Sci., NS-30, p.3499, 1983.

[8] H. Frischolz, W. Schnell, IEEE Trans Nucl. Sci., Nㅗ-24, p. 1683, 1977. 